\title{
Colloid monolayer lithography-A flexible approach for nanostructuring of surfaces
}

\author{
F. Burmeister *, W. Badowsky, T. Braun, S. Wieprich, J. Boneberg, P. Leiderer \\ Department of Physics, University of Constance, D-78434 Constance, Germany
}

\begin{abstract}
In this paper, we report on an alternative nanofabrication method which relies on self-assembly of colloidal particles into a two-dimensional array on surfaces. Some important parameters for obtaining large monolayers of good crystallinity are discussed, i.e., the evaporation rate of the colloid suspension, the surface charge of the particles and the wetting properties of the substrate. The 2D crystals can be utilized as lithographic masks for consecutive processes. This is demonstrated with two experiments, site-selective electrochemical deposition of copper on a semiconductor surface and shape modification of small metallic dots by thermal annealing. 1999 Elsevier Science B.V. All rights reserved.
\end{abstract}

PACS: $82.70 . \mathrm{D} ; 61.46 ; 81.40 . \mathrm{E}$

Keywords: Nanostructuring; Self-assembly; Colloids; Metal dots

\section{Introduction}

More and more companies and research institutes concentrate on 'nanotechnology' as the technology for future markets. Therefore, basic research on nanostructures becomes increasingly important. It has already been shown that nanostructured materials and surfaces with dimensions below $100 \mathrm{~nm}$ exhibit new physical properties, e.g., optical, catalytic and magnetic [1]. Conventional techniques, especially electron-beam and ion-beam lithography, are in principle capable of producing structures with dimensions far below $100 \mathrm{~nm}$, i.e., it should be possible to fabricate such nanostructures and study their properties. However, besides some practical limits, these

\footnotetext{
* Corresponding author. Tel.: +49-7531-88-2065; Fax.: +497531-3127; E-mail: frank.burmeister@uni-konstanz.de
}

methods are serial and so cost-consuming that only few laboratories can afford them.

Alternatively, many recent research activities have been devoted to parallel techniques, which are based on self-organising processes [2]. One of these methods, lithography with colloid monolayers [3-5], will be discussed in this paper. By some means (e.g., solvent evaporation, spin coating or electrophoresis $[6,7]$ ), a hexagonally close-packed monolayer of equally sized spherical particles is fabricated on a substrate and serves as a mask for consecutive processes like vacuum deposition or ion etching. The obtainable structure size is only limited by the size of the spherical particles, which can be produced with narrow size distributions down to several tens of nanometers. The technique has already found several applications [4,8], and its underlying physical processes are well understood. Nevertheless, the 
technique and its potentials are not very common among the scientific community.

We will first discuss the production method for colloid monolayers which is based on the evaporation of the dispersion medium. In Section 2, we present two possible applications of colloid monolayer lithography, both in combination with other methods.

\section{Fabrication of $2 \mathrm{D}$ crystals}

All experiments were carried out with commercially available sulphatized polystyrene spheres (IDC, Portland, OR) with various sizes from $110 \mathrm{~nm}$ to 3 $\mu \mathrm{m}$ and polydispersities ranging from 1.7 to $5.5 \%$. All particles were charge-stabilized and, according to the manufacturer, free of additional surfactants. They were diluted with Milli-Q water without further purification.

For the monolayer production of close-packed spheres, we followed the approach of Micheletto et al. [9]: first, a droplet of a colloidal suspension is applied onto the substrate. Then, it is mounted onto a peltier element for temperature control inside a closed box. The box is slightly tilted against the horizontal and the peltier element holds the substrate temperature roughly $1^{\circ} \mathrm{C}$ above the ambient temperature. After complete evaporation of the dispersion medium, polycrystalline mono- and multilayers of the colloidal particles, usually polystyrene spheres, are left behind. According to Denkov et al. [10], the crystal formation proceeds via two steps: first, a crystal nucleus is formed due to capillary forces between neighbouring particles, which protrude from the solvent surface. Further solvent evaporation and capillary rise of the menisci between adjacent spheres causes water influx from the surrounding and more particles are transported towards the nucleus.

We will now discuss some physical and chemical parameters that determine the quality and size of the obtained arrays (see also Ref. [9]). Important physical parameters are (1) the inclination angle of the box towards the horizontal and (2) the evaporation rate of the dispersion medium. The basic idea behind the inclination of the box is a deformation of the suspension droplet such that nucleation starts at the upper edge of the droplet and crystal growth pro- ceeds downwards in a defined direction. The droplet deformation gives a lower limit of the inclination angle. An upper limit is given by the following: in the case of a contact angle hystereses, capillary forces compete with gravity and a droplet can remain stuck on a tilted surface without sliding [11].

Between these two limits, which were 3 and $15^{\circ}$ in our experiments, we could not find any dependence of crystal quality and size on the tilt angle: for these investigations, we built two identical closed boxes, where the evaporation of the colloidal suspension droplets took place. In this way, we could directly compare colloidal monolayers fabricated as identically as possible, where only the inclination angle was varied. Afterwards, the fraction of the sample covered with a monolayer as well as average crystallite size was determined by electron or optical microscopy. This was done for various combinations of particle sizes and substrate materials.

Another physical parameter is the evaporation rate of the dispersion medium which is assumed to be water from here on. It is directly proportional to the vapour pressure and depends exponentially on temperature: $p \sim \exp \left(-W / k_{\mathrm{B}} T\right)$, where $W$ denotes the molar evaporation energy. In general, lower evaporation rates, i.e., lower temperatures, favours larger crystallites. As this dependency was extensively studied in Ref. [12], we only report on our additional video microscopy observations: during the crystal growth, particles in the arrays leading edge are still mobile for several seconds such that freshly formed microcrystallites reorient themselves to fit into an already existing larger domain (see Fig. 1). The temporal distance between two images is $0.1 \mathrm{~s}$. Rapid evaporation shortens the interval during which the particles are mobile and leads to smaller crystallites.

Next, we investigated the chemical parameters which we deemed important, (1) the chemical homogeneity and the wetting properties of the substrate and (2) the concentration and the properties of the particles themselves. Several authors have already pointed out the importance of a clean and hydrophillic substrate $[8,9,13]$. Having tried out at least five different cleaning procedures for glass substrates, we employed one based on an Ethanol/ $\mathrm{NaOH}$-solution, which leaves a surface terminated with $\mathrm{OH}^{-}$-groups [14]. The contact angle of a watery 

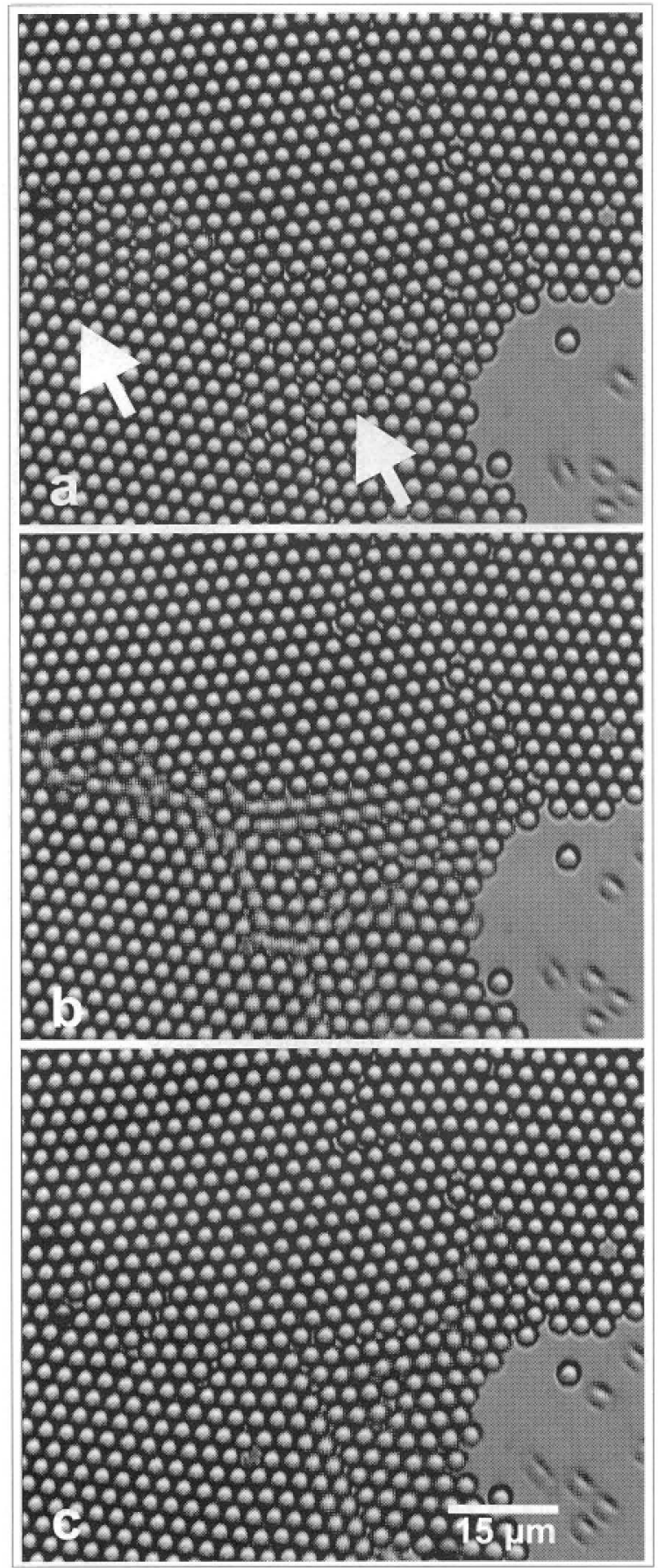

Fig. 1. Optical microscope images of $3-\mu \mathrm{m}$ spheres during crystal growth. Note the reorientation of the marked areas. suspension on such a surface is close to zero. Only with glass slides prepared in this way, we managed to prepare $\mathrm{cm}^{2}$-sized monolayers of particles down to $170 \mathrm{~nm}$ in diameter (in the special case of particles smaller than optical wavelengths, the areas of the substrate which were covered by monolayers could be easily identified due to their distinct interference colours [15]). With even smaller particles (110 and $64 \mathrm{~nm}$ ), we only obtained stripes of particle deposits with equal proportions of mono- and multilayers. This might be due to a rupture of the thin water film in the last stage of drying and agrees with ellipsometry measurements of Dushkin et al. [16] and Adachi et al. [17]. They did not observe stable water films on glass below a thickness of $120 \mathrm{~nm}$.

Another important aspect is the following: the surface termination of the glass substrate with $\mathrm{OH}^{-}$groups causes a negative surface charge in water such that the negatively charged, sulphatized spheres are repelled from the surface and maintain their mobility even in very thin films. On the other hand, experiments with positively charged spheres, e.g., with amidine groups on their surface, revealed that nucleus formation due to capillary forces still takes place to some extent, whereas convective influx of particles and therefore crystal growth was completely hindered. In Fig. 2a, one only sees disordered groups of particles neighboured by particle-free regions. After coating the substrates with a $10 \mathrm{~nm}$ thick layer of $\mathrm{Al}_{2} \mathrm{O}_{3}$, which is positively charged in water, we obtained the same results as with sulphatized spheres on negatively charged glass slides, namely a well-ordered, hexagonal array (Fig. 2b).

The last parameter we want to discuss is the concentration of the suspension. Knowing the volume of the suspension droplet, one can easily calculate the theoretical concentration one needs to cover a given area with one monolayer of spheres [9]. However, as a competitive process to $2 \mathrm{D}$ crystal growth, we always observed multilayer particle deposits at the edge of the suspension droplet, similar to coffee stains. Again, the physical origin of this rim is a pronounced curvature of the droplet at the contact line which leads to an increased evaporation rate [18]. Consecutive water influx from inside the droplet transports particles towards the rim. This process decreases the number of particles left for monolayer formation. Therefore, the actual concen- 
tration of the colloidal suspension should always be higher than the one calculated. On the other hand, concentrations much larger than the theoretical ones led to multilayer growth. Using two to three times the concentration as the theoretical one turned out to give the best results in our experiments (at least 20 for each sphere size).

In summary, the fabrication of well-ordered colloidal monolayers is complicated by an interplay of several physical and chemical parameters. whose

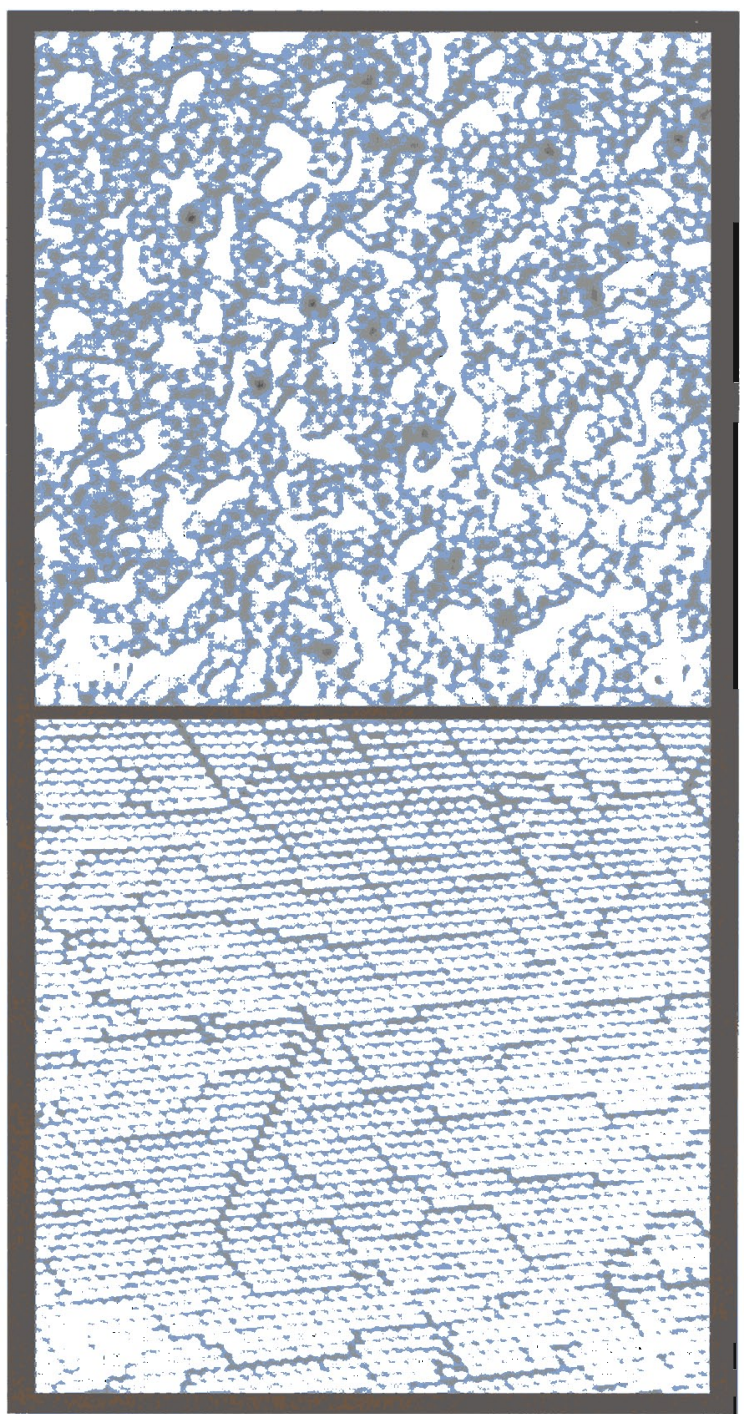

Fig. 2. Optical microscope pictures of dried monolayers of $810-\mathrm{mm}$ spheres on glass which was (a) $\mathrm{OH}^{-}$-terminated, (b) $\mathrm{Al}_{2} \mathrm{O}_{7}$-coated.

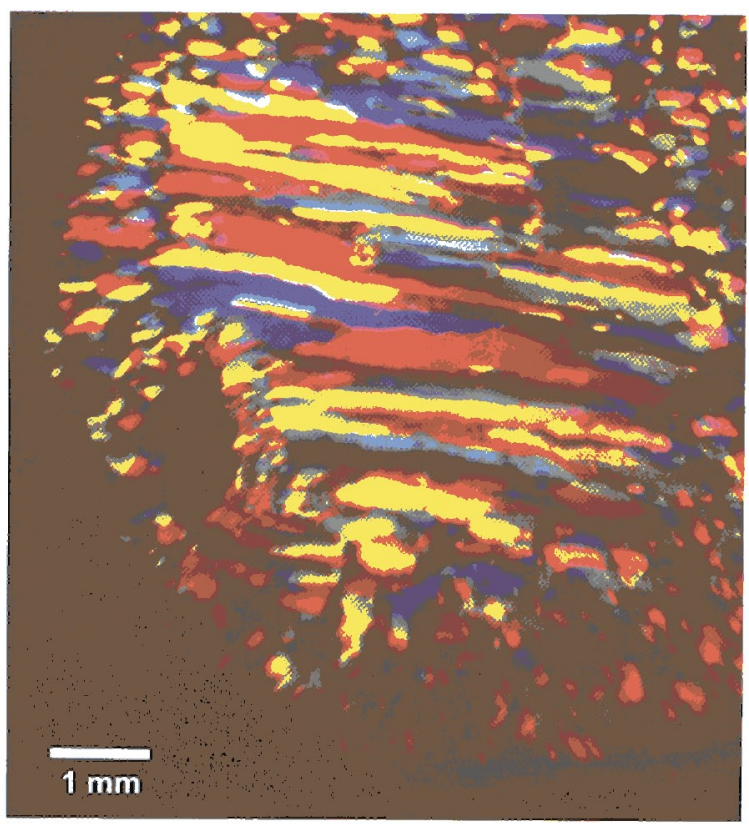

Fig. 3. Photograph of a monolayer of $1.7-\mu \mathrm{m}$ spheres illuminated with white light (seen in reflection mode).

influence is often difficult to distinguish. After considering all parameters mentioned above, we have managed to fabricate monolayers with crystallites containing up to $10^{6}$ particles (see Fig. 3). 'There, a monolayer of $1.7-\mu \mathrm{m}$ particles on silicon is illuminated with white light. The different colours are due to Bragg-reflections of differently oriented domains. The elongation of the uniformly coloured stripes also indicates the direction of the array growth. Highermagnification optical microscopy still revealed point defects like missing particles but a uniform orientation of the particles throughout the grains.

\subsection{Applications}

An example of how colloid monolayers can be used to fabricate well-defined nanostructures is

\footnotetext{
${ }^{1}$ The area of the largest stripes lies around $1-2 \mathrm{~mm}^{2}$. A hexagonally closed-packed layer covers about $90 \%$ of the area, i.e., $0.9-1.8 \mathrm{~mm}^{2}$. This number, divided by the area covered by one particle. $1.7 \mu \mathrm{m}^{2} \times \pi / 4$, gives $0.5-1 \times 10^{6}$ particles per grain as a rough estimate.
} 
shown in Fig. 4. We first prepared a monolayer film of 840-nm particles on a glass slide, floated it off and transferred it onto the surface of the layered semiconductor $\mathrm{WSe}_{2}$ [13]. In a second step, the sample was bombarded with argon ions with an energy of $1 \mathrm{keV}$. The particles protected the underlying substrate such that only material underneath the particle interstices was removed (Fig. 4a). Afterwards, the particles were lift off in an ultrasonic bath. The van-der-Waals-surfaces of layered materials like semiconducting $\mathrm{WSe}_{2}$ are chemically inert, whereas the crystal faces perpendicular to the vander-Waals-plane, which have been created during the ion bombardment exhibit dangling bonds [19]. We

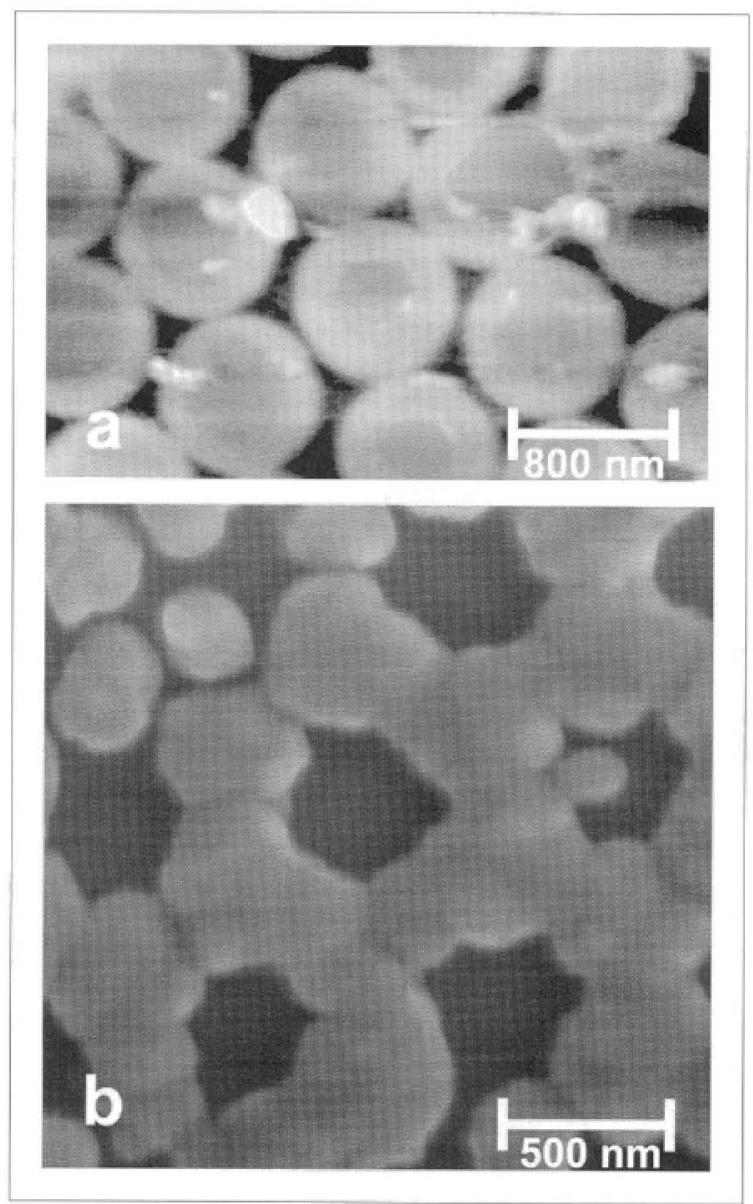

Fig. 4. WSe ${ }_{2}$-surface after ion-bombardment through colloid monolayer and removal of spheres, (a) AFM picture, depth of trenches $10 \mathrm{~nm}$, (b) SEM picture after electrochemical copper deposition.

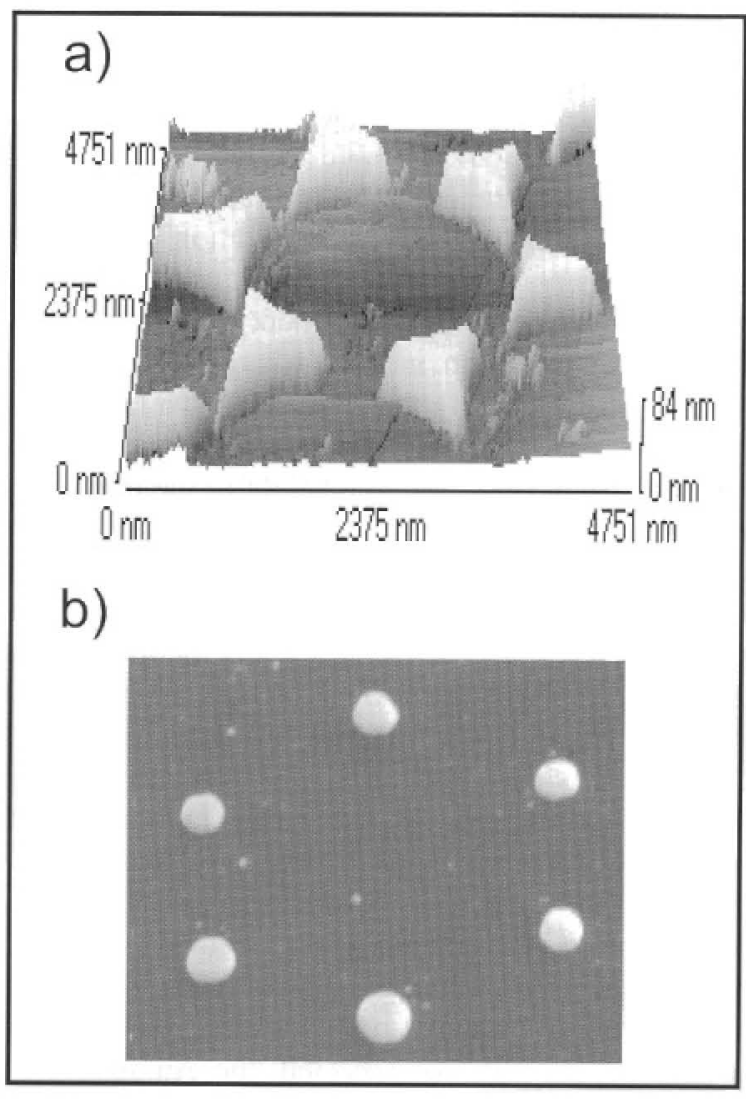

Fig. 5. Au structures on HOPG after thermal evaporation through colloid monolayer of $3-\mu \mathrm{m}$ spheres, (a) AFM picture, (b) after annealing for $1 \mathrm{~h}$ at $800^{\circ} \mathrm{C}, \mathrm{SEM}$ picture. Note the compressed scale in $y$-direction due to the oblique view angle.

then used the structured surface as a working electrode in a typical three-electrode setup. The electrolyte was a $0.1 \mathrm{~mol} \mathrm{H}_{2} \mathrm{SO}_{4} / 0.05 \mathrm{~mol} \mathrm{CuSO}_{4}$-solution and a copper wire served as a counter electrode. A Ag/ $\mathrm{AgCl}$-electrode was used as a reference electrode in a separate compartment of the electrochemical cell. The application of a potential slightly more negative than the redox potential of copper $(337 \mathrm{mV}$ vs. NHE) led to copper deposition onto the surface. The deposition took place preferentially at the damaged areas of the sample (Fig. 4b). As we also observed preferential adsorption on natural defects, like steps and dislocations, we believe that the defects created by the ion bombardment, served as nucleation sites for deposited atoms. This may be compared with UHV experiments, where similar findings were made [20]. 
Although we have performed only some preliminary experiments so far, we assume that, depending on the voltage sweep conditions and electrolyte concentration, one should be able to fabricate not only isolated copper islands as before, but also metallic networks on inert substrates. In addition, the whole process does not need any high-vacuum equipment.

The second kind of experiment also deals with metallic structures on inert substrates. Again, with the help of the floating technique [13], we fabricated triangular $\mathrm{Au}$ structures on graphite with a side length of $1 \mu \mathrm{m}$ (Fig. 5a). The height of the triangular gold structures was $60 \mathrm{~nm}$ with a typical island structure on top. They were then annealed for $1 \mathrm{~h}$ under argon flow at a temperature of $800^{\circ} \mathrm{C}$, in order to modify the shape of the metallic structures. Such shape modifications are known to occur upon annealing of evaporated Au films on a graphite substrate [21] as well as upon annealing of $\mathrm{Au}$ islands on mica [22]. They can be attributed to the high cohesion energy of $\mathrm{Au}$ compared to the interface energy, which favours the formation of a quasispherical shape with plane facets upon reaching equilibrium [21]. Fig. 5b shows a scanning electron microscope picture of the resulting Au particles after such an annealing experiment. As expected, the Au structures exhibit a quasispherical shape. During the annealing process, the height of the structures has been increased, whereas their diameter has been reduced to $450 \mathrm{~nm}$ due to volume conservation. The annealing of nanostructures can therefore be used to produce monodisperse, spherical nanoparticles of almost arbitrary diameter at well defined distances by a suitable choice of the height of the evaporated structures.

\section{Conclusion and outlook}

With the help of two examples, we have discussed possible applications of colloid monolayer lithography. Various physical and chemical parameters, which determine crystal quality and size, were investigated. The combination of colloid monolayer lithography with electrochemistry or thermal annealing opens up new possibilities for the creation of non-triangular structures like metallic networks or single erystallites. It also demonstrates the possibility of fabricating structures much smaller than dictated by the sphere size, e.g., just by lowering the electrolyte concentration.

\section{Acknowledgements}

This work was financed by the Deutsche Forschungsgemeinschaft, SFB 513.

\section{References}

[1] D.J. Wales, Science 271 (1996) 925, all review articles in Feb. issue of Science 96.

[2] Z.L. Wang, Adv. Mater, 10 (1998) 13

[3] H.W. Deckman, J.H. Dunsmuir, Appl. Phys. Lett. 41 (1982) 377.

[4] U.Ch. Fischer, H.P. Zingsheim, J. Vac. Sci. Technol. 19 (1981) 881 .

[5] J.C. Hulteen, R.P. Van Duyne, J. Vac. Sci. Technol. 13 (1995) 1553.

[6] F. Burmeister, C. Schäfle, B. Keilhofer, C. Bechinger, J. Boneberg, P. Leiderer, Adv. Mater. 10 (1998) 495.

[7] M. Trau, D.A. Saville, I.A. Aksay, Science 272 (1996) 706.

[8] P.D. Vu, J.R. Olson, R.O. Pohl, Ann. Phys. 4 (1995) 9.

[9] R. Micheletto, H. Fukuda, M. Ohtsu, Langmuir 11 (1995) 3333.

[10] N.D. Denkov, O.D. Velev, P.A. Kralchevsky, I.B. Ivanov, H. Yoshimura, K. Nagayama, Langmuir 8 (1992) 3183.

[11] D. Quere, M.-J. Azzopardi, L. Delattre, Langmuir 14 (1998) 2213.

[12] S. Rakers, L.F. Chi, H. Fuchs, Langmuir 13 (1997) 7121.

[13] F. Burmeister, C. Schäfle, T. Matthes, M. Böhmisch, J. Boneberg. P. Leiderer, Langmuir 13 (1997) 2983.

[14] Z. Yang, H. Yu, Adv. Mater. 9 (1997) 426.

[15] C.D. Dushkin, K. Nagayama, T. Miwa, P.A. Kralchevsky, Langmuir 9 (1993) 3695.

[16] C.D. Dushkin, H. Yoshimura, K. Nagayama, Chem. Phys. Lett. 204 (1993) 455.

[17] E. Adachi, A.S. Dimitrov, K. Nagayama, Langmuir 11 (1995) 1057.

[18] R.D. Deegan, O. Bakajin, T.F. Dupont, G. Huber, S.R. Nagel, T.A. Witten, Nature 389 (1997) 827.

[19] A. Aruchamy, Photoelectrochemistry and Photovoltaics of Layered Semiconductors, Kluwer, London, 1992.

[20] A. Rettenberger, P. Bruker, M. Metzler, F. Mugele, Th.W. Matthes, M. Böhmisch, J. Boneberg, K. Friemelt, P. Leiderer, Surf. Sci. $402-404$ (1996) 409.

[21] J.C. Heyraud, J.J. Metois, J. Cryst. Growth 50 (1980) 571.

[22] L.F. Chi, T. Drechsler, St. Höppener, S. Rakers, Ch. Röthig, Th. Schwaack, F. Starrberg, H. Fuchs, J. Surf, Anal. 3 (1998) 168. 